\title{
Mental health status and quality of life in elderly patients with coronary heart disease
}

\author{
Min Tang ${ }^{\text {Equal first author, } 1}{ }^{1}$, Song-Hao Wang Equal first author, ${ }^{1}$, Hui-Lin $\mathrm{Li}^{1}{ }^{1}$, Han Chen ${ }^{1}$, Xin-Yi Sun ${ }^{1}$, Wei-Wei Bian ${ }^{2}$, Jing \\ Sheng ${ }^{\text {Corresp., } 1}$, Shao-Jun Ma ${ }^{\text {Corresp. } 1}$ \\ ${ }^{1}$ Department of Geriatrics $\square$ Shanghai Ninth People's Hospital, Shanghai Jiao Tong University School of Medicine, Shanghai, China \\ 2 Department of Plastic and Reconstructive Surgery, Shanghai Ninth People's Hospital, Shanghai Jiao Tong University School of Medicine, Shanghai, China \\ Corresponding Authors: Jing Sheng, Shao-Jun Ma \\ Email address: shengjing60@163.com, mashj@163.com
}

Background. Coronary heart disease (CHD) is the leading cause of morbidity and mortality among elderly individuals. Patients with CHD are at high risk for mental health disorders, and psychological issues may affect the quality of life (QoL) of these patients. Nevertheless, there is little evidence regarding the psychological issues and QoL of patients with CHD among the elderly population. This study aimed to investigate the relationship between comorbidities and mental status as well as QoL among elderly patients with CHD. Methods. Overall, 216 patients were included in this cross-sectional, observational, single-center study. The demographics and clinical manifestations of the patients were collected from electronic medical records. All patients were interviewed using the Chinese version of Symptom Checklist 90 (SCL-90) to assess the mental health status and the World Health Organization Quality of Life-BREF questionnaire ( WHOQOLBREF ) to assess the QoL. Results. In total, 96 men and 120 women, with a mean age of $71.69 \pm 8.30$ years, were included. When controlling for the patients' sex, marital status and stroke, multiple stepwise linear regression analyses suggested that for CHD patients, comorbid type 2 diabetes mellitus had the significant influence on average positive factors (Coef., 5.809; 95\% Cl: 2.467, 9.152; $p=0.001$ ); when controlling for the patients' sex, marital status and type 2 diabetes mellitus, multiple stepwise linear regression analyses suggested that for CHD patients, comorbid stroke had the significant influence on average positive factors (Coef., 8.680; 95\% Cl: 4.826, 12.534; $p<0.001$ ); when controlling for the patients' sex, marital status, type 2 diabetes mellitus and stroke, multiple stepwise linear regression analyses suggested that for CHD patients, comorbid primary hypertension had the significant influence on phobic anxiety (Coef., $0.178 ; 95 \% \mathrm{Cl}: 0.010,0.347 ; p=0.038$ ).

Conclusions. For elderly CHD patients, comorbid type 2 diabetes mellitus and stroke were at risk for psychological problems and lower QoL. Our findings may guide patients and clinicians to make better decisions and achieve better outcomes. 
1 Mental health status and quality of life in elderly

2 patients with coronary heart disease

3

4

5

6

7

8

9

10

11

12

13

14

Min Tang ${ }^{1 a}$, Song-Hao Wang ${ }^{1 a}$, Hui-Lin Li ${ }^{1}$, Han Chen ${ }^{1}$, Xin-Yi Sun ${ }^{1}$, Wei-Wei Bian², Jing Sheng ${ }^{1 *}$, Shao-Jun $\mathrm{Ma}^{1 *}$

1'Department of Geriatrics, Shanghai Ninth People's Hospital, Shanghai Jiao Tong University School of Medicine: No. 639, Zhizaoju Road, Shanghai 200011, China.

2Department of Plastic and Reconstructive Surgery, Shanghai Ninth People's Hospital, Shanghai Jiao Tong University School of Medicine: No. 639, Zhizaoju Road, Shanghai 200011, China.

*Corresponding author at: Department of Geriatrics, Shanghai Ninth People's Hospital, Shanghai Jiao Tong University School of Medicine: No. 639, Zhizaoju Road, Shanghai 200011, China. E-mail address: Shao-Jun Ma, mashj@163.com; Jing Sheng, shengjing60@163.com a These authors contributed equally to this work.

\section{Abstract}

Background. Coronary heart disease (CHD) is the leading cause of morbidity and mortality among elderly individuals. Patients with CHD are at high risk for mental health disorders, and psychological issues may affect the quality of life (QoL) of these patients. Nevertheless, there is little evidence regarding the psychological issues and QoL of patients with CHD among the elderly population. This study aimed to investigate the relationship between comorbidities and mental status as well as QoL among elderly patients with CHD.

Methods. Overall, 216 patients were included in this cross-sectional, observational, single-center study. The demographics and clinical manifestations of the patients were collected from electronic medical records. All patients were interviewed using the Chinese version of Symptom Checklist 90 (SCL-90) to assess the mental health status and the World Health Organization Quality of Life-BREF questionnaire (WHOQOL-BREF) to assess the QoL.

Results. In total, 96 men and 120 women, with a mean age of $71.69 \pm 8.30$ years, were included. When controlling for the patients' sex, marital status and stroke, multiple stepwise linear regression analyses suggested that for CHD patients, comorbid type 2 diabetes mellitus had the significant influence on average positive factors (Coef., 5.809; 95\% CI: 2.467, 9.152; $p=0.001$ ); when controlling for the patients' sex, marital status and type 2 diabetes mellitus, multiple stepwise linear regression analyses suggested that for CHD patients, comorbid stroke had the significant influence on average positive factors (Coef., 8.680; 95\% CI: 4.826, 12.534; $p$ $<0.001$ ); when controlling for the patients' sex, marital status, type 2 diabetes mellitus and 
41 stroke, multiple stepwise linear regression analyses suggested that for CHD patients, comorbid

42 primary hypertension had the significant influence on phobic anxiety (Coef., 0.178; 95\% CI:

$430.010,0.347 ; p=0.038)$.

44 Conclusions. For elderly CHD patients, comorbid type 2 diabetes mellitus and stroke were at 45 risk for psychological problems and lower QoL. Our findings may guide patients and clinicians 46 to make better decisions and achieve better outcomes.

47

\section{Introduction}

49 Coronary heart disease (CHD) is the leading cause of death worldwide(Wang et al. 2016). The 50 incidence and prevalence of CHD increase greatly with age(Kattainen et al. 2006). Therefore, 51 CHD is the major cause of morbidity and mortality among elderly individuals, which contributes to a substantial economic burden on the healthcare system(Hanna \& Wenger 2005). Patients with CHD are at high risk for mental health disorders, such as depression and anxiety(Bashiri et al. 2016), which have both been strongly related to adverse outcomes in patients with CHD(Barth et al. 2004). These psychological problems may not only increase the use of healthcare services,

56

57

58

59

60

61

62

63

64

65

66

67

68

69

70

71

72

73

74

75

76

77

78

79

80 but they also result in disease deterioration(Dehdari et al. 2009). In addition, CHD had a severe impact on the quality of life (QoL)(Dyer et al. 2010). A previous study has shown that most of the elderly population has three or more comorbid illnesses(Caughey et al. 2008). As people age, the risk of chronic conditions increases, such as diabetes, heart disease, cancer and arthritis( $\mathrm{Wu}$ et al. 2013). Previous research suggested a higher incidence of coexisting diseases in elderly patients, and cardiovascular comorbidities and metabolic diseases were commonly encountered diseases(Man in't Veld 1998; Van den Akker et al. 1998).Comorbidity is related to a decline in many health outcomes including QoL and psychological distress, which increases the use of health care resources(Fortin et al. 2006a; Fortin et al. 2006b; Wolff et al. 2002). However, until now, there have been few studies that investigated the psychological issues and QoL of elderly CHD patients, and the psychological states and QoL of elderly CHD patients are not clear. Therefore, correlational studies are needed.

The SCL-90 is a widely used, self-rating questionnaire that contains multidimensional subscales to measure overall health status. This scale contains 90 items, using 10 factors, to evaluate the psychiatric symptoms in 10 aspects, which is regarded as a widely used psychological measurement in China and several studies have assessed the validity of this instrument(Zhao et al. 2019). The SCL-90 contains a very broad range of psychiatric symptomatology involving feelings, emotion, thinking, consciousness, behavior, lifestyle, interpersonal relationships, diet and sleep(Wei et al. 2018).

QoL is an increasingly important health issue in healthcare intervention(Al-Taie et al. 2019). The WHOQOL-BREF is a short version of the WHOQOL-100, which is a rapid tool for the assessment of QoL in clinical studies(Skevington et al. 2004). The properties of this questionnaire have been tested and verified for evaluation in diverse cultural groups(Skevington 
81 et al. 2004), which has suggested that it is a valid assessment with higher cross-cultural

82

83

84

85

86

87

88

89

90

91

92

93

94

95

96

97

98 99

100

101

102

103

104

105

106

107

108

109

110

111

112

113

114

115

116

117

118

119

120

applicability and it is widely recognized.

Patient self-rating health status measurements may be a useful tool to screen high-risk patients to target for more efficient preventative measures, interventions and treatments in clinical practice(Spertus et al. 2002). Due to the adequate reliability and validity of above two scales, we used a Chinese version of SCL-90 to assess the mental health status and the WHOQOL-BREF questionnaire to assess the QoL in patients with CHD, and we conducted a cross-sectional study to investigate the relationship between the comorbidities and mental health status as well as QoL among elderly patients with CHD.

\section{Materials \& Methods}

\section{Study Design}

This cross-sectional, observational, single-center study was designed to investigate the association between the type of comorbidities and mental status as well as QoL among elderly patients with CHD. This study was approved by the China Ethics Committee of Registering Clinical Trials (number, ChiECRCT-2017046) and registered with the Chinese Clinical Trial Registry (number, ChiCTR1900021276). Informed consent was obtained from all patients.

\section{Study setting and subjects}

All subjects of this study were screened as inpatients who were hospitalized in the Department of Cardiology and Geriatrics at Shanghai Ninth People's Hospital, Shanghai Jiao Tong University School of Medicine, between February 2017 and January 2018. A total of 216 patients with a prior diagnosis of CHD (International Classification of Diseases 10: codes I20-I25) were enrolled. The inclusion criteria were those aged $\geqslant 60$ years, with a prior diagnosis of $\mathrm{CHD}$. The exclusion criteria were patients aged under 60 years, those with organic psychosis, those who refused to give informed consent and who were unable to complete the scale. When patients of the study were hospitalized more than once during the study period, we only used the first record of that time to avoid double counting.

\section{Procedures and assessment methods}

The purpose of the cross-sectional trial was to assess the mental health status and QoL in elderly patients with CHD. A face-to-face interview was performed for each patient in a private meeting room. All interviews were made by the same doctor who received related training. All patients were interviewed using the Chinese version of SCL-90 to assess the mental health status, and the WHOQOL-BREF questionnaire assessed QoL. The demographic characteristics and clinical manifestations of the patients, such as age, gender, height, weight, body mass index (BMI), marital status and concomitant diseases, were collected from electronic medical records, and these characteristics were verified by the patients during the interview. 
121 SCL-90 was comprised of 90 items which consisted of 10 factors, including somatization (12 122 items), obsessive-compulsive (10 items), interpersonal sensitivity ( 9 items), depression (13 123 items), anxiety (10 items), hostility (6 items), phobic anxiety (7 items), paranoid ideation (6 124 items), psychoticism (10 items) and other factors (sleep and diet, 7 items). Each item was scored 125 using a 5 -point scale $(0=$ none, $1=$ slight, $2=$ mild, $3=$ moderate, $4=$ severe). This questionnaire 126 indicated a positive item was with a score of more than 2, which may suggest potential mental 127 health issues for that aspect. A higher score of average positive factors indicated a more severe 128 psychological problem(Wei et al. 2018).

129

The WHOQOL-BREF is a widely used tool that has adequate reliability and validity to assess

131

132

133

134

135

136

137

138

139

140

141

142

143

144

145

146

147

148

149

150

151

152

153

154

155

156

157

158

159

160 the QoL. The WHOQOL-BREF questionnaire has 26 items including two general items and 24 items, and 24 items to evaluate four main domains of the QoL, which referred to the physical status(7 items), psychological health(6 items), social relations(3 items), and environmental factors(8 items)(Ohaeri \& Awadalla 2009). Each item is scored using a five-point scale ranging from 1 (very dissatisfied or very poor) to 5 (very satisfied or very good). Mean scores of items are multiplied by four to make domain scores ranging from 4 to 20 . And scores of the two general items also range from 4 to 20 , respectively.

\section{Study variables}

The parameters, including demographic characteristics, clinical manifestations, concomitant comorbidities and the scores of the two assessment scales, were evaluated in the 216 patients with CHD. CHD with comorbidity of primary hypertension, type 2 diabetes mellitus and stroke, respectively, was analyzed. A set of the following clinical parameters was analyzed: age, sex, height, weight, BMI, marital status, presence of primary hypertension, presence of type 2 diabetes mellitus, presence of stroke, average positive factors and nine factors of SCL-90 scale, the scores of four domains of the WHOQOL-BREF scale.

\section{Statistical analysis}

Data were shown as number (\%) or as mean (standard deviation). The $t$ test was used for the continuous variables. The associations were examined using multiple stepwise linear regression analyses, and variables including age, sex, BMI, marital status, primary hypertension, type 2 diabetes mellitus and stroke were entered into the multiple stepwise linear regression. We used multiple stepwise linear regression analyses to generate significant fit models. The coefficients and $95 \%$ confidence interval $(\mathrm{CI})$ for each significant variable were determined. A $p$-value of $<0.05$ was considered statistically significant. Statistical analyses were performed using IBM SPSS 24.0.

\section{Results}

In total, 96 men and 120 women, with a mean age of 71.69(8.30) years, were included. Among the 216 patients, 209 (96.8\%) patients were married, and 7 (3.2\%) patients were widowed or 
161 bachelors. Patients had a mean height of $165.80(8.27) \mathrm{cm}$, a mean weight of 70.78(9.01) kg, and

162

163

164

165

166

167

168

169

170

171

172

173

174

175

176

177

178

179

180

181

182

183

184

185

186

187

188

189

190

191

192

193

194

195

196

197

198

199

200 a mean BMI of $25.71(2.32) \mathrm{kg} / \mathrm{m}^{2}$. The demographic characteristics of elderly patients with CHD are presented in Table 1.

In univariate analysis, the mean number of positive factors were $26.18(14.88)$ and 20.69 (9.54) for participants with and without primary hypertension, respectively ( $p=0.003)$; the mean scores of WHOQOL-BREF physical domain were 12.43 (2.82) and 13.58 (2.08) for participants with and without primary hypertension, respectively $(p=0.003)$. The mean number of positive factors were 29.54 (14.97) and 22.23 (12.79) for participants with and without type 2 diabetes mellitus, respectively $(p<0.001)$; the mean scores of WHOQOL-BREF physical domain were 11.74 (2.90) and 13.25 (2.44) for participants with and without type 2 diabetes mellitus, respectively ( $p$ $<0.001)$; the mean scores of WHOQOL-BREF psychological domain were 12.93 (2.42) and 13.85 (2.22) for participants with and without type 2 diabetes mellitus, respectively $(p=0.005)$. The mean number of positive factors were 33.24 (15.66) and 22.57 (12.63) for participants with and without stroke, respectively $(p<0.001)$; the mean scores of WHOQOL-BREF physical domain were 11.31 (2.96) and 13.08 (2.51) for participants with and without stroke, respectively $(p<0.001)$; the mean scores of WHOQOL-BREF psychological domain were $12.84(2.72)$ and 13.70 (2.18) for participants with and without stroke, respectively $(p=0.022)$. The results of univariate analyses are shown in Supplementary Tables 1, 2, and 3.

Multiple stepwise linear regression analyses suggested that for CHD patients, when controlling for the patients' sex, marital status and stroke, comorbid type 2 diabetes mellitus had the significant influence on average positive factors (Coef., 5.809; 95\% CI: 2.467, 9.152; $p=0.001$ ), interpersonal sensitivity (Coef., $0.151 ; 95 \%$ CI: 0.033, 0.270; $p=0.012$ ), depression (Coef., 0.241; 95\% CI: 0.100, 0.381; $p=0.001$ ), anxiety (Coef., 0.151; 95\% CI: 0.035, 0.268; $p=0.011$ ), psychoticism (Coef., 0.146; 95\% CI: 0.045, 0.247; $p=0.005$ ), physical domain (Coef., -1.272; 95\% CI: $-1.953,-0.591 ; p<0.001)$; when controlling for the patients' sex and stroke, comorbid type 2 diabetes mellitus had the significant influence on somatization (Coef., 0.346 ; $95 \% \mathrm{CI}$ : $0.159,0.533 ; p<0.001)$; when controlling for the patients' age, marital status and stroke, comorbid type 2 diabetes mellitus had the significant influence on hostility (Coef., $0.128 ; 95 \%$ CI: $0.015,0.242 ; p=0.027$ ); when controlling for the patients' sex, marital status, stroke and primary hypertension, comorbid type 2 diabetes mellitus had the significant influence on phobic anxiety (Coef., 0.165; 95\% CI: 0.025, 0.305; $p=0.021$ ); when controlling for the patients' marital status and stroke, comorbid type 2 diabetes mellitus had the significant influence on paranoid ideation (Coef., 0.097; 95\% CI: 0.003, 0.192; $p=0.044$ ); when controlling for the patients' sex and marital status, comorbid type 2 diabetes mellitus had the significant influence on psychological domain (Coef., -0.855 ; 95\% CI: $-1.477,-0.234 ; p=0.007$ ).

Multiple stepwise linear regression analyses suggested that for CHD patients, when controlling for the patients' sex, marital status and type 2 diabetes mellitus, comorbid stroke had the

Peer) reviewing PDF | (2020:08:52224:4:0:NEW 8 Jan 2021) 
201 significant influence on average positive factors (Coef., 8.680; 95\% CI: 4.826, 12.534; $p$

$202<0.001$ ), interpersonal sensitivity (Coef., 0.232 ; 95\% CI: 0.096, 0.368; $p=0.001$ ), depression

203 (Coef., 0.401; 95\% CI: 0.239, 0.563; $p<0.001$ ), anxiety (Coef., 0.143; 95\% CI: 0.009, 0.278;

$204 p=0.037$ ), psychoticism (Coef., 0.305; 95\% CI: 0.189, 0.422; $p<0.001$ ), physical domain (Coef., $205-1.416$; 95\% CI: $-2.202,-0.631 ; p<0.001)$; when controlling for the patients' sex and type 2

206 diabetes mellitus, comorbid stroke had the significant influence on somatization (Coef., 0.356;

207 95\% CI: 0.141, 0.572; $p=0.001$ ); when controlling for the patients' sex and marital status,

208 comorbid stroke had the significant influence on obsessive-compulsive (Coef., 0.308; 95\% CI:

$2090.162,0.453 ; p<0.001$ ); when controlling for the patients' age, marital status and type 2 diabetes

210 mellitus, comorbid stroke had the significant influence on hostility (Coef., $0.168 ; 95 \%$ CI: 0.032 ,

$2110.304 ; p=0.016$ ); when controlling for the patients' sex, marital status, type 2 diabetes mellitus

212 and primary hypertension, comorbid stroke had the significant influence on phobic anxiety

213 (Coef., 0.320; 95\% CI: 0.162, 0.479; $p<0.001$ ); when controlling for the patients' marital status

214 and type 2 diabetes mellitus, comorbid stroke had the significant influence on paranoid ideation

215 (Coef., 0.214; 95\% CI: 0.105, 0.323; $p<0.001$ );

216

217 When controlling for the patients' sex, marital status, type 2 diabetes mellitus and stroke,

218 multiple stepwise linear regression analyses suggested that for CHD patients, comorbid primary

219 hypertension had the significant influence on phobic anxiety (Coef., $0.178 ; 95 \%$ CI: 0.010 ,

$2200.347 ; p=0.038$ ). The relationship between scores of the two scales and three comorbidities is

221 presented in Tables 2 and 3.

222

\section{Discussion}

224 Our findings suggested that for CHD patients, comorbid type 2 diabetes mellitus was positively

225

226

227

228

229

230

231

232

233

234

235 associated with higher scores in average positive factors, somatization, interpersonal sensitivity, depression, anxiety, hostility, phobic anxiety, paranoid ideation and psychoticism, respectively; while comorbid stroke was positively associated with higher scores in average positive factors, somatization, obsessive-compulsive, interpersonal sensitivity, depression, anxiety, hostility, phobic anxiety, paranoid ideation, psychoticism, respectively; comorbid primary hypertension was positively associated with higher scores in phobic anxiety; higher scores represented potential mental health issues. Therefore, the presence of these comorbidities resulted in poorer mental health among elderly patients with CHD. On the other hand, for CHD patients, comorbid type 2 diabetes mellitus was negatively associated with scores of the physical and psychological domain; comorbid stroke was negatively associated with scores of the physical domain. Our

236

237

238 results suggested that with comorbidity of these comorbidities resulted in a lower QoL among elderly patients with CHD. To the best of our knowledge, this is the first study to examine the association between the type of comorbidities and mental health status and QoL among elderly patients with CHD.

239 
240 A previous study reported that co-existing psychological problems were more harmful than a 241 single psychological problem for patients with several basic diseases(Pincus et al. 2004).

242 Multidimensional psychological assessment can aid clinicians to comprehensively and better

243 understand the symptoms and mental health status of patients(Helzer et al. 2009). In our study,

244 we used the SCL-90 scale to assess psychological status, which was commonly used in

245 psychiatric and psychological counseling clinics to evaluate the psychological statuses and

246 mental issues among people having different occupations(Wei et al. 2018). Several previous

247 studies demonstrated a link between depression and CHD(Reid et al. 2013), diabetes(Goins et al.

248 2019), as well as age(Reid et al. 2013). Besides, mental health disorders are common in patients

249 with stroke(Almeida \& Xiao 2007). Similarly, our findings demonstrated that for elderly CHD

250 patients, comorbid type 2 diabetes mellitus or stroke was associated with a higher SCL-90 score;

251 however, further studies with larger samples of patients are necessary to verify our findings.

252 Additionally, in this population, hypertension influenced the factor of phobic anxiety, which may

253 be a useful basis for further research as few studies focus on the comorbidities of elderly

254 patients. Being informed of mental health status might be useful in screening mental health

255 needs, which can guide health care interventions(Moriarty et al. 2009). Our study adopted the

256 multidimensional to assess the different aspects of mental health in elderly CHD patients with

257 comorbidities, which may be a useful basis for clinical practice.

258

259

260

CHD had a severe impact on human health and QoL(Dyer et al. 2010); therefore, QoL was an

261 important predictor of health outcomes in CHD treatment(Cepeda-Valery et al. 2011). CHD patients had a lower QoL, which may be affected by psychological symptoms(Raymakers et al.

262

263 2018; Saengsiri et al. 2014). Besides, published studies indicated that comorbidities may affect the elderly patients' QoL(Jacobs 2009; Rambod et al. 2020), and our study indicated that for elderly CHD patients, comorbid type 2 diabetes mellitus was negatively associated with the scores of the WHOQOL-BREF physical and psychological domains. Therefore, comorbid type 2 diabetes mellitus negatively affected the QoL, which was consistent with previous studies(Marfella et al. 2018; Sardu et al. 2019). A published study showed that cognitive function was a predictor of the physical function domain of health-related QoL(Patel et al. 2007). Also, psychological problems, such as anxiety and depression, are common in stroke patients(De Wit et al. 2017; Froes et al. 2011). Furthermore, our study indicated that comorbid stroke was negatively associated with the scores of the WHOQOL-BREF physical domain, which was consistent with previous studies. In addition, our study suggested that for elderly CHD patients, comorbid primary hypertension did not significantly influence the scores of WHOQOL-BREF domains. Comorbidity was an important factor in health-related QoL deterioration associated with aging, and it should be noted that comorbidity can affect different domains of QoL to

276 varying degrees(Zygmuntowicz et al. 2012). Our findings may serve as a piece of evidence in 277 health care. 
279 There are some limitations to our study. First, our study was limited by its observational nature.

280 The data were from a single hospital, and the sample was relatively small. Second, this was a

281 cross-sectional study in which we identified the relationship between the scores of two scales and

282 the type of comorbidities; however, longitudinal studies are needed to examine the relationship

283 over time(AlRuthia et al. 2020). Finally, this is a questionnaire-based survey, and the results

284 might have been negatively affected by acquiescence bias, social desirability bias, and

285 interviewer bias(Bowling 2005). Moreover, there might have been recall bias in elderly patients

286 with a decline in memory. The self-rating questionnaire may result in an additional form of bias

287 (Murphy et al. 2014).

288

289

\section{Conclusions}

290

In this study, we studied the association between the types of comorbidities among elderly

291 patients with CHD and mental health and QoL. The results suggested that for elderly CHD

292 patients, comorbid type 2 diabetes mellitus were more likely to exist with psychological

293 problems and lower QoL than the population without type 2 diabetes mellitus. In addition, our

294 study suggested that comorbid stroke affected mental health and QoL in the target population.

295 These findings may guide patients and clinicians to make better decisions and help this

296 population to achieve better outcomes.

297

298

\section{Acknowledgements}

299

We are grateful to all the participants who took part in this study.

300

301

\section{Disclosure Statement}

302

The authors have no conflicts of interest to declare.

303

304

\section{Funding Sources}

305

The authors received no funding for this work.

306

307

308

309

310

\section{Data Availability}

The following information was supplied regarding data availability: the raw data has been supplied as Data S1 and Data S2.

\section{References}

Al-Taie N, Maftei D, Kautzky-Willer A, Krebs M, and Stingl H. 2019. Assessing the quality of life among patients with diabetes in Austria and the correlation between glycemic control and the quality of life. Primary Care Diabetes.

Almeida OP, and Xiao J. 2007. Mortality associated with incident mental health disorders after stroke. Aust N Z J Psychiatry 41:274-281. 10.1080/00048670601172772

AlRuthia Y, Sales I, Almalag H, Alwhaibi M, Almosabhi L, Albassam AA, Alharbi FA, Bashatah A, and Asiri Y. 2020. The Relationship Between Health-Related Quality of Life and Trust 
319

320

321

322

323

324

325

326

327

328

329

330

331

332

333

334

335

336

337

338

339

340

341

342

343

344

345

346

347

348

349

350

351

352

353

354

355

356

357

358

359

360

361

362

363

364

365

366

367

368

369 in Primary Care Physicians Among Patients with Diabetes. Clinical Epidemiology 12:143.

Barth J, Schumacher M, and Herrmann-Lingen C. 2004. Depression as a risk factor for mortality in patients with coronary heart disease: a meta-analysis. Psychosom Med 66:802-813. 10.1097/01.psy.0000146332.53619.b2

Bashiri Z, Aghajani M, and Masoudi Alavi N. 2016. Effects of Psychoeducation on Mental Health in Patients With Coronary Heart Disease. Iran Red Crescent Med J 18:e25089. 10.5812/ircmj.25089

Bowling A. 2005. Mode of questionnaire administration can have serious effects on data quality. Journal of public health 27:281-291.

Caughey GE, Vitry Al, Gilbert AL, and Roughead EE. 2008. Prevalence of comorbidity of chronic diseases in Australia. BMC Public Health 8:221. 10.1186/1471-2458-8-221

Cepeda-Valery B, Cheong AP, Lee A, and Yan BP. 2011. Measuring health related quality of life in coronary heart disease: the importance of feeling well. Int J Cardiol 149:4-9. 10.1016/j.ijcard.2010.09.048

De Wit L, Theuns P, Dejaeger E, Devos S, Gantenbein AR, Kerckhofs E, Schuback B, Schupp W, and Putman K. 2017. Long-term impact of stroke on patients' health-related quality of life. Disabil Rehabil 39:1435-1440. 10.1080/09638288.2016.1200676

Dehdari T, Heidarnia A, Ramezankhani A, Sadeghian S, and Ghofranipour F. 2009. Effects of progressive muscular relaxation training on quality of life in anxious patients after coronary artery bypass graft surgery. Indian J Med Res 129:603-608.

Dyer MT, Goldsmith KA, Sharples LS, and Buxton MJ. 2010. A review of health utilities using the EQ-5D in studies of cardiovascular disease. Health and quality of life outcomes 8:13.

Fortin M, Bravo G, Hudon C, Lapointe L, Almirall J, Dubois MF, and Vanasse A. 2006a. Relationship between multimorbidity and health-related quality of life of patients in primary care. Qual Life Res 15:83-91. 10.1007/s11136-005-8661-z

Fortin M, Bravo G, Hudon C, Lapointe L, Dubois MF, and Almirall J. 2006b. Psychological distress and multimorbidity in primary care. Ann Fam Med 4:417-422. 10.1370/afm.528

Froes KS, Valdes MT, Lopes Dde P, and Silva CE. 2011. Factors associated with health-related quality of life for adults with stroke sequelae. Arq Neuropsiquiatr 69:371-376. 10.1590/s0004-282x2011000300020

Goins RT, Noonan C, Winchester B, and Brock D. 2019. Depressive Symptoms and All-Cause Mortality in Older American Indians with Type 2 Diabetes Mellitus. J Am Geriatr Soc 67:1940-1945. 10.1111/jgs. 16108

Hanna IR, and Wenger NK. 2005. Secondary prevention of coronary heart disease in elderly patients. Am Fam Physician 71:2289-2296.

Helzer JE, Kraemer HC, Krueger RF, Wittchen H-U, Sirovatka PJ, and Regier DA. 2009. Dimensional approaches in diagnostic classification: Refining the research agenda for $D S M-V$ : American Psychiatric Pub.

Jacobs J. 2009. Quality of life: what does it mean for general practice? : British Journal of General Practice.

Kattainen A, Salomaa V, Harkanen T, Jula A, Kaaja R, Kesaniemi YA, Kahonen M, Moilanen L, Nieminen MS, Aromaa A, and Reunanen A. 2006. Coronary heart disease: from a disease of middle-aged men in the late 1970s to a disease of elderly women in the 2000s. Eur Heart J 27:296-301. 10.1093/eurheartj/ehi630

Kruithof N, Haagsma JA, Karabatzakis M, Cnossen MC, de Munter L, van de Ree CLP, de Jongh MAC, and Polinder S. 2018. Validation and reliability of the Abbreviated World Health Organization Quality of Life Instrument (WHOQOL-BREF) in the hospitalized trauma population. Injury 49:1796-1804. 10.1016/j.injury.2018.08.016

Man in't Veld A. 1998. Symptomatic BPH and hypertention: Does comorbidity affect quality of life? European urology 34:29-36. 
370

371

372

373

374

375

376

377

378

379

380

381

382

383

384

385

386

387

388

389

390

391

392

393

394

395

396

397

398

399

400

401

402

403

404

405

406

407

408

409

410

411

412

413

414

415

416

417

418

419

Marfella R, Sardu C, Calabro P, Siniscalchi M, Minicucci F, Signoriello G, Balestrieri ML, Mauro C, Rizzo MR, Paolisso G, and Barbieri M. 2018. Non-ST-elevation myocardial infarction outcomes in patients with type 2 diabetes with non-obstructive coronary artery stenosis: Effects of incretin treatment. Diabetes Obes Metab 20:723-729. 10.1111/dom.13122

Moriarty DG, Zack MM, Holt JB, Chapman DP, and Safran MA. 2009. Geographic patterns of frequent mental distress: U.S. adults, 1993-2001 and 2003-2006. Am J Prev Med 36:497-505. 10.1016/j.amepre.2009.01.038

Murphy G, Snetselaar L, Myers E, LaForge B, Qualls LG, Hand RK, Blackwelder S, and Bradley D. 2014. Self-reported health parameters compared with clinician measurements: methods in practice-based research. J Public Health Manag Pract 20:513-522. 10.1097/PHH.0b013e3182a998fd

Ohaeri JU, and Awadalla AW. 2009. The reliability and validity of the short version of the WHO Quality of Life Instrument in an Arab general population. Ann Saudi Med 29:98-104. 10.4103/0256-4947.51790

Patel MD, McKevitt C, Lawrence E, Rudd AG, and Wolfe CD. 2007. Clinical determinants of long-term quality of life after stroke. Age Ageing 36:316-322. 10.1093/ageing/afm014

Pincus HA, Tew JD, and First MB. 2004. Psychiatric comorbidity: is more less? World Psychiatry 3:18-23.

Rambod M, Ghodsbin F, and Moradi A. 2020. The Association Between Body Mass Index and Comorbidity, Quality of Life, and Cognitive Function in the Elderly Population. International Journal of Community Based Nursing \& Midwifery 8:45-54.

Raymakers AJN, Gillespie P, Murphy E, Cupples ME, Smith SM, Murphy AW, Griffin MD, Benyamini $Y$, and Byrne M. 2018. Patient reported health status and all-cause mortality in patients with coronary heart disease. Fam Pract 35:172-178. 10.1093/fampra/cmx094

Reid J, Ski CF, and Thompson DR. 2013. Psychological interventions for patients with coronary heart disease and their partners: a systematic review. PLoS One 8:e73459. 10.1371/journal.pone.0073459

Saengsiri A-o, Thanasilp S, and Preechawong S. 2014. Factors predicting quality of life for coronary artery disease patients after percutaneous coronary intervention. Asian Biomedicine 8:31-42.

Sardu C, Paolisso P, Sacra C, Mauro C, Minicucci F, Portoghese M, Rizzo MR, Barbieri M, Sasso FC, D'Onofrio N, Balestrieri ML, Calabro P, Paolisso G, and Marfella R. 2019. Effects of Metformin Therapy on Coronary Endothelial Dysfunction in Patients With Prediabetes With Stable Angina and Nonobstructive Coronary Artery Stenosis: The CODYCE Multicenter Prospective Study. Diabetes Care 42:1946-1955. 10.2337/dc182356

Skevington SM, Lotfy M, O'Connell KA, and Group W. 2004. The World Health Organization's WHOQOL-BREF quality of life assessment: psychometric properties and results of the international field trial. A report from the WHOQOL group. Qual Life Res 13:299-310. 10.1023/B:QURE.0000018486.91360.00

Spertus JA, Jones P, McDonell M, Fan V, and Fihn SD. 2002. Health status predicts long-term outcome in outpatients with coronary disease. Circulation 106:43-49. 10.1161/01.cir.0000020688.24874.90

Van den Akker M, Buntinx F, Metsemakers JF, Roos S, and Knottnerus JA. 1998. Multimorbidity in general practice: prevalence, incidence, and determinants of co-occurring chronic and recurrent diseases. Journal of clinical epidemiology 51:367-375.

Wang H, Naghavi M, Allen C, Barber RM, Bhutta ZA, Carter A, Casey DC, Charlson FJ, Chen $A Z$, and Coates MM. 2016. Global, regional, and national life expectancy, all-cause mortality, and cause-specific mortality for 249 causes of death, 1980-2015: a systematic analysis for the Global Burden of Disease Study 2015. The lancet 388:1459-1544.

PeerJ reviewing PDF | (2020:08:52224:4:0:NEW 8 Jan 2021) 
420

421

422

423

424

425

426

427

428

429

430

431

432

433

434

435

436
Wei Y, Li H, Wang H, Zhang S, and Sun Y. 2018. Psychological Status of Volunteers in a Phase I Clinical Trial Assessed by Symptom Checklist 90 (SCL-90) and Eysenck Personality Questionnaire (EPQ). Med Sci Monit 24:4968-4973. 10.12659/MSM.909524

Wolff JL, Starfield B, and Anderson G. 2002. Prevalence, expenditures, and complications of multiple chronic conditions in the elderly. Arch Intern Med 162:2269-2276. 10.1001/archinte.162.20.2269

Wu F, Guo Y, Kowal P, Jiang Y, Yu M, Li X, Zheng Y, and Xu J. 2013. Prevalence of major chronic conditions among older Chinese adults: the Study on Global AGEing and adult health (SAGE) wave 1. Plos One 8.

Zhao M, Feng Z, and Yang G. 2019. Improvement of mental health among Chinese plateau military personnel, 1993-2017: a cross-temporal meta-analysis of the Symptom Checklist-90. Neuropsychiatr Dis Treat 15:2971-2980. 10.2147/NDT.S202088

Zygmuntowicz M, Owczarek A, Elibol A, and Chudek J. 2012. Comorbidities and the quality of life in hypertensive patients. Pol Arch Med Wewn 122:333-340. 


\section{Table $\mathbf{1}$ (on next page)}

Table 1. Demographic characteristics of the elderly patients with $\mathrm{CHD}(\mathrm{N}=216)$ 
1 Mental Health Status and Quality of Life in Elderly Patients with Coronary Heart Disease

3 Table 1. Demographic characteristics of the elderly patients with $\mathrm{CHD}(\mathrm{N}=\mathbf{2 1 6})$

\begin{tabular}{ll}
\hline Demographic characteristic & Number \\
\hline Age(years), mean (SD) & $71.69(8.30)$ \\
Height (cm), mean (SD) & $165.80(8.27)$ \\
Weight $(\mathrm{kg})$, mean (SD) & $70.78(9.01)$ \\
BMI $\left(\mathrm{kg} / \mathrm{m}^{2}\right)$, mean (SD) & $25.71(2.32)$ \\
Gender & \\
Male, N (\%) & $96(44.4)$ \\
Female, N (\%) & $120(55.6)$ \\
Marital status & $209(96.8)$ \\
Married, N (\%) & $7(3.2)$ \\
Widowed or bachelor, N (\%)
\end{tabular}

4 Abbreviation: N-number

5 


\section{Table 2 (on next page)}

Table 2. Multiple linear regression analysis of the association between SCL-90 factors and three comorbidities in elderly patients with $\mathrm{CHD}(\mathrm{N}=216)$ 
1 Table 2. Multiple linear regression analysis of the association between SCL-90 factors and three comorbidities in elderly patients with CHD

$2 \quad(\mathrm{~N}=216)$

3

\begin{tabular}{|c|c|c|c|c|c|c|c|c|c|c|}
\hline \multirow{2}{*}{ Variable } & \multicolumn{2}{|c|}{ Average positive factors } & \multicolumn{2}{|c|}{ Somatization } & \multicolumn{2}{|c|}{ Obsessive-compulsive } & \multicolumn{2}{|c|}{ Interpersonal sensitivity } & \multicolumn{2}{|c|}{ Depression } \\
\hline & Coef. (95\% C.I.) & p value & Coef. (95\% C.I.) & p value & Coef. (95\% C.I.) & p value & Coef. (95\% C.I.) & p value & Coef. (95\% C.I.) & p value \\
\hline Sex & $8.355(5.096 ; 11.614)$ & $<0.001$ & $0.418(0.236 ; 0.601)$ & $<0.001$ & $0.275(0.152 ; 0.399)$ & $<0.001$ & $0.162(0.047 ; 0.278)$ & 0.006 & $0.243(0.106 ; 0.379)$ & 0.001 \\
\hline Marital status & $-20.357(-29.442 ;-$ & $<0.001$ & - & - & $-0.718(-1.064 ;-0.373)$ & $<0.001$ & $-0.482(-0.803 ;-$ & 0.003 & $-0.827(-1.208 ;-$ & $<0.001$ \\
\hline Type 2 DM & $5.809(2.467 ; 9.152)$ & 0.001 & $0.346(0.159 ; 0.533)$ & $<0.001$ & - & - & $0.151(0.033 ; 0.270)$ & 0.012 & $0.241(0.100 ; 0.381)$ & 0.001 \\
\hline Stroke & $8.680(4.826 ; 12.534)$ & $<0.001$ & $0.356(0.141 ; 0.572)$ & 0.001 & $0.308(0.162 ; 0.453)$ & $<0.001$ & $0.232(0.096 ; 0.368)$ & 0.001 & $0.401(0.239 ; 0.563)$ & $<0.001$ \\
\hline
\end{tabular}

4

\begin{tabular}{|c|c|c|c|c|c|c|c|c|c|c|}
\hline \multirow{2}{*}{ Variable } & \multicolumn{2}{|l|}{ Anxiety } & \multicolumn{2}{|l|}{ Hostility } & \multicolumn{2}{|c|}{ Phobic anxiety } & \multicolumn{2}{|c|}{ Paranoid ideation } & \multicolumn{2}{|l|}{ Psychoticism } \\
\hline & Coef. (95\% C.I.) & p value & Coef. (95\% C.I.) & p value & Coef. (95\% C.I.) & p value & Coef. (95\% C.I.) & p value & Coef. (95\% C.I.) & $p$ \\
\hline Sex & $0.243(0.130 ; 0.357)$ & $<0.001$ & - & - & $0.215(0.083 ; 0.348)$ & 0.002 & - & - & $0.181(0.082 ; 0.279)$ & $<0.00$ \\
\hline Marital status & $-0.713(-1.030 ;-0.396)$ & $<0.001$ & $-0.356(-0.667 ;-$ & 0.025 & $-0.512(-0.881 ;-0.142)$ & 0.007 & $-0.558(-0.816 ;-0.299)$ & $<0.001$ & $-0.496(-0.770 ;-0.222)$ & $<0.00$ \\
\hline Age (years) & - & - & $-0.011(-0.018 ;-$ & 0.002 & - & - & - & - & - & - \\
\hline Primary & - & - & - & - & $0.178(0.010 ; 0.347)$ & 0.038 & - & - & - & - \\
\hline Type 2 DM & $0.151(0.035 ; 0.268)$ & 0.011 & $0.128(0.015 ; 0.242)$ & 0.027 & $0.165(0.025 ; 0.305)$ & 0.021 & $0.097(0.003 ; 0.192)$ & 0.044 & $0.146(0.045 ; 0.247)$ & 0.005 \\
\hline Stroke & $0.143(0.009 ; 0.278)$ & 0.037 & $0.168(0.032 ; 0.304)$ & 0.016 & $0.320(0.162 ; 0.479)$ & $<0.001$ & $0.214(0.105 ; 0.323)$ & $<0.001$ & $0.305(0.189 ; 0.422)$ & $<0.00$ \\
\hline
\end{tabular}

5 Abbreviations: Coef. - Coefficient; C.I. - confidence interval; Type 2 DM- type 2 diabetes mellitus

6 Reference: Sex-male; Marital status- widowed or bachelor; Primary hypertension-no primary hypertension; Type 2 DM-no type 2 DM; Stroke-no stroke 


\section{Table 3(on next page)}

Table 3. Multiple linear regression analysis of the association between WHOQOL-BREF domains and three comorbidities in elderly patients with $\mathrm{CHD}(\mathrm{N}=216)$ 
1 Table 3. Multiple linear regression analysis of the association between WHOQOL-BREF domains and three comorbidities in elderly patients with

2 CHD $(\mathbf{N}=\mathbf{2 1 6})$

3

\begin{tabular}{|c|c|c|c|c|c|c|c|c|c|}
\hline \multirow{2}{*}{ Variable } & \multirow{2}{*}{ Reference } & \multicolumn{2}{|l|}{ Physical } & \multicolumn{2}{|l|}{ Psychological } & \multicolumn{2}{|l|}{ Social } & \multicolumn{2}{|c|}{ Environment } \\
\hline & & Coef. (95\% C.I.) & p value & Coef. (95\% C.I.) & p value & Coef. (95\% C.I.) & p value & Coef. (95\% C.I.) & p value \\
\hline Stroke & No stroke & $-1.416(-2.202 ;-$ & $<0.001$ & - & - & - & - & - & - \\
\hline Type 2 DM & No type 2 DM & $-1.272(-1.953 ;-$ & $<0.001$ & $-0.855(-1.477 ;-0.234)$ & 0.007 & - & - & - & - \\
\hline Sex & Male & $-1.244(-1.908 ;-$ & $<0.001$ & $-0.930(-1.539 ;-0.321)$ & 0.003 & - & - & $-0.625(-1.091 ;-$ & 0.009 \\
\hline Marital & Widowed or & $2.662(0.811 ; 4.513)$ & 0.005 & $1.847(0.145 ; 3.548)$ & 0.034 & - & - & - & - \\
\hline
\end{tabular}

4 Abbreviations: Coef. - Coefficient; C.I. - confidence interval; Type 2 DM- type 2 diabetes mellitus

5

6

7

8 